01

\title{
Моделирование некоторых свойств изображений с атомарным разрешением в сканирующем зондовом микроскопе
}

\author{
(C) A.А. Потапов, ${ }^{1,3,4}$ C.Ш. Рехвиашвили ${ }^{2, q}$ \\ ${ }^{1}$ Институт радиотехники и электроники им. В.А. Котельникова РАН, \\ 125009 Москва, Россия \\ ${ }^{2}$ Институт прикладной математики и автоматизации КБНЦ РАН, \\ 360000 Нальчик, Россия \\ ${ }^{3}$ JiNan University (JNU), \\ Guangzhou, China \\ ${ }^{4}$ Cooperative Chinese-Russian laboratory of informational technologies and signals fractal processing of JNU-IREE RAS, \\ JiNan University (JNU), \\ Guangzhou, China \\ ^e-mail: rsergo@mail.ru
}

(Поступило в Редакцию 9 января 2017 г. В окончательной редакции 7 декабря 2017 г.)

Разработана методика моделирования изображений в сканирующем зондовом микроскопе (СЗМ) с одновременным применением вейвлет-преобразования и медианной фильтрации. В работе использовалось вейвлет-преобразование с ядром Добеши 4-го порядка. Данное преобразование позволяет выделять на СЗМ-изображениях детали различных масштабов, что дает возможность исследовать фрактальные свойства поверхностей. С помощью моделирования показано, что сверхвысокое разрешение в СЗМ может соответствовать атомарному, если размеры контактной области в системе „зонд-образец“ намного превышает атомные размеры, а также имеется случайный разброс в расположении атомов решетки. Дано объяснение явлению инверсии контраста на СЗМ-изображениях в режиме многократного сканирования.

DOI: $10.21883 /$ JTF.2018.06.46008.2159

\section{Введение}

В настоящее время мультимодовые сканирующие зондовые микроскопы (СЗМ) насчитывают десятки модификаций - от простейших учебных приборов до сложных сверхвысоковакуумных нанотехнологических комплексов, которые включают в себя сканирующий туннельный микроскоп (СТМ), атомно-силовой микроскоп (АСМ), сканирующий ближнепольный микроскоп (СБОМ), прецизионную оптику, молекулярно-лучевую эпитаксию, технику ионных и электронных пучков и др.

При практическом использовании СЗМ приходится сталкиваться с различными искажениями электрических сигналов - артефактами. Все артефакты в СЗМ можно разделить на два типа: приборные и физические. К приборным артефактам относятся перекрестные помехи, нелинейность сканера, запаздывание и старение пьезокерамики, creер-эффект, шумы в электрических схемах. Для их устранения используют программные и аппаратные средства коррекции. Физические артефакты обусловлены конкретными физическими явлениями в системе „зонд-образец“. К ним, в частности, можно отнести флуктуации туннельного тока [1], эффект двойной иглы [2,3], интерференция от зонда, капиллярное смачивание контакта [4]. В большинстве случаев физические артефакты устраняются изменением условий эксперимента, например изменением рабочих напряжений или токов, помещением измерительной головки в вакуумную камеру, сменой зонда и т. п.
Для изучения особенностей формирования изображений в СЗМ с атомарным уровнем разрешения в режимах АСМ и СТМ широко используется численное моделирование [5-11]. При этом для расчета силы взаимодействия между зондом и образцом производится суммирование парного межатомного потенциала взаимодействия; расчет туннельного тока учитывает волновые функции или структурно-зависимые электронные состояния зонда и образца. В настоящей работе предлагается формализованная математическая модель, в рамках которой предполагается, что результирующий сигнал (сила взаимодействия в АСМ или туннельный ток в СТМ) в системе регистрации СЗМ пропорционален количеству атомов, попадающих в зону контакта зонда и образца с кристаллической структурой поверхности. Для обработки получаемых изображений предлагается одновременно использовать вейвлет-преобразование и медианное сглаживание. Данная математическая модель позволяет исследовать такие важные факторы, как появление атомарного разрешения, влияние дефектов решетки, усиление или ослабление контраста изображений.

\section{Математическая модель}

Будем предполагать, что в СЗМ при сближении зонда с кристаллическим образцом образуется плоская контактная область, которая представляет собой окружность радиусом $R$ (рис. 1). Эта окружность в процессе сканирования перемещается вдоль поверхности и заме- 


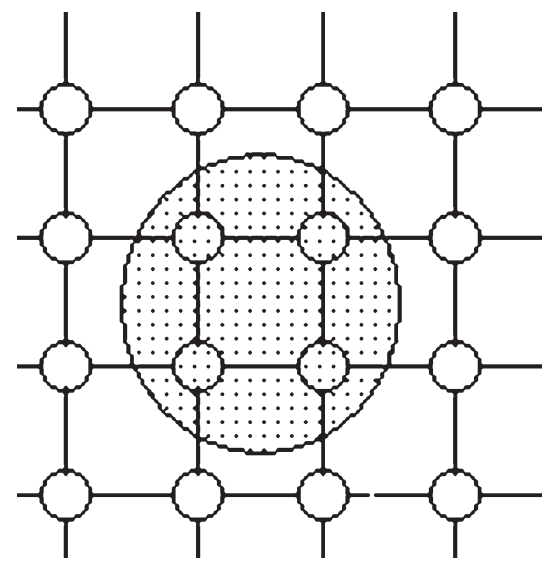

Рис. 1. Модель контакта в СЗМ.

тает некоторую часть атомов решетки, от количества которых зависит результирующий сигнал при формировании изображения. Число атомов внутри окружности равно

$$
\begin{gathered}
N(x, y)=\sum_{i} \sum_{j} \Phi\left(R^{2}-\left(x-x_{i}\right)^{2}-\left(y-y_{j}\right)^{2}\right) . \\
\Phi(x)= \begin{cases}0, & x<0, \\
1, & x \geq 0,\end{cases}
\end{gathered}
$$

где $x$ и $y$ - координаты центра окружности, соответствующие координатам зонда при сканировании, $x_{i}$ и $y_{j}$ - координаты узлов решетки, соответствующие атомам образца, $\Phi(x)$ - функция Хевисайда.

В работе [12] для обработки изображений и анализа физических процессов, происходящих в системе „зонд-образец“ АСМ, впервые предложено использовать непрерывное вейвлет-преобразование. Было показано, что вейвлет-преобразование позволяет эффективно решать задачу о подавлении шума на модельных АСМизображениях. В настоящей работе для аналогичных целей предлагается использовать дискретное вейвлетпреобразование с ядром Добеши 4-го прядка $[13,14]$ :

$$
\begin{gathered}
f(t)=\sum_{j, k} c_{j, k} \psi_{j, k}(t), \\
\psi_{j, k}(t)=2^{j / 2} \psi\left(2^{j} t-k\right), \quad j, k \in I . \\
c_{j, k}=W\left(\frac{1}{2^{j}}, \frac{k}{2^{j}}\right), \quad W(a, b)=\frac{1}{a} \int_{-\infty}^{\infty} u(t) \psi\left(\frac{t-b}{a}\right) d t,
\end{gathered}
$$

где $W(a, b)$ - функция, определяющая вейвлет-спектр, переменные $a$ и $b$ задают расширение и сдвиг для покрытия сигнала $t(t)$ базовым вейвлетом. Функция $\psi(x)$ выражается через коэффициенты Добеши [13,14]. Для покрытия всего сигнала короткими вейвлетами в (2) используются процедуры сдвига и масштабного преобразования. В итоге сигнал представляется совокупностью параметрических „вейвлетных волн“, зависящих от частоты (масштаба) и координаты (сдвига). Как известно, основное преимущество вейвлет-преобразования перед фурье-преобразованием заключается в том, что оно позволяет эффективно обрабатывать сложные нерегулярные сигналы, следить за изменениями спектральных характеристик со временем и определять, какие частоты и масштабы преобладают в исследуемом сигнале.

В теории вейвлет-преобразования доказывается аналог теоремы Парсерваля, поэтому можно считать, что энергия сигнала пропорциональна квадрату соответствующего коэффициента разложения: $E \sim W^{2}(a, b)$. В связи с этим для подавления шума на СЗМ-изображениях можно использовать процедуру редактирования вейвлетспектра, которая в нашем случае осуществляется следующим образом:

$$
\tilde{W}(a, b)=W(a, b) \Phi( \pm|W(a, b)| \mp \Delta),
$$

где $\Delta-$ уровень выделения спектра. Знаки в аргументе функции Хевисайда выбираются в зависимости от необходимости выделения нужных деталей в изображении: нижний знак ставится для выделения мелкомасштабных деталей в сигнале, а верхний - для выделения крупномасштабных деталей. В результате замены $W(a, b)$ на $\tilde{W}(a, b)$ в (2) получается отфильтрованный сигнал.

Для сглаживания помех, имеющих импульсный характер, нами выбрано медианное сглаживание (см. подробно [15]). Этот выбор обусловлен тем, что метод медианного сглаживания имеется в программном обеспечении современных СЗМ [16]. Суть метода заключается в выборе скользящего окна, которое охватывает нечетное количество точек изображения $N$. Центральная точка заменяется медианой всех точек в окне. При этом медианой для нечетного $N$ является элемент, для которого существуют $(N-1) / 2$ элементов, равных или меньших

$$
G:=\mid \begin{aligned}
& \text { for } \mathrm{i} \in 0 \ldots \mathrm{N} \\
& \qquad \begin{array}{l}
\text { for } \mathrm{j} \in 0 . . \mathrm{N} \\
\mathrm{p} 1_{\mathrm{j}} \leftarrow \mathrm{M}_{\mathrm{i}, \mathrm{j}} \\
\mathrm{pw} \leftarrow \operatorname{wave}(\mathrm{p} 1) \\
\text { for } \mathrm{j} \in 0 . . \mathrm{N} \\
\mathrm{pw} \mathrm{w}_{\mathrm{j}} \leftarrow \mathrm{pw} \mathrm{w}_{\mathrm{j}} \cdot \Phi\left(\left|\mathrm{pw} \mathrm{w}_{\mathrm{j}}\right|-\Delta\right) \\
\mathrm{p} 1 \leftarrow \mathrm{iwave}(\mathrm{pw}) \\
\mathrm{p} 2 \leftarrow \operatorname{medsmooth}(\mathrm{p} 1,5) \\
\text { for } \mathrm{j} \in 0 \ldots \mathrm{N} \\
\mathrm{p} 3_{\mathrm{i}, \mathrm{j}} \leftarrow \mathrm{p} 2_{\mathrm{j}}
\end{array}
\end{aligned}
$$

Рис. 2. Фрагмент программы моделирования изображений в СЗМ. 

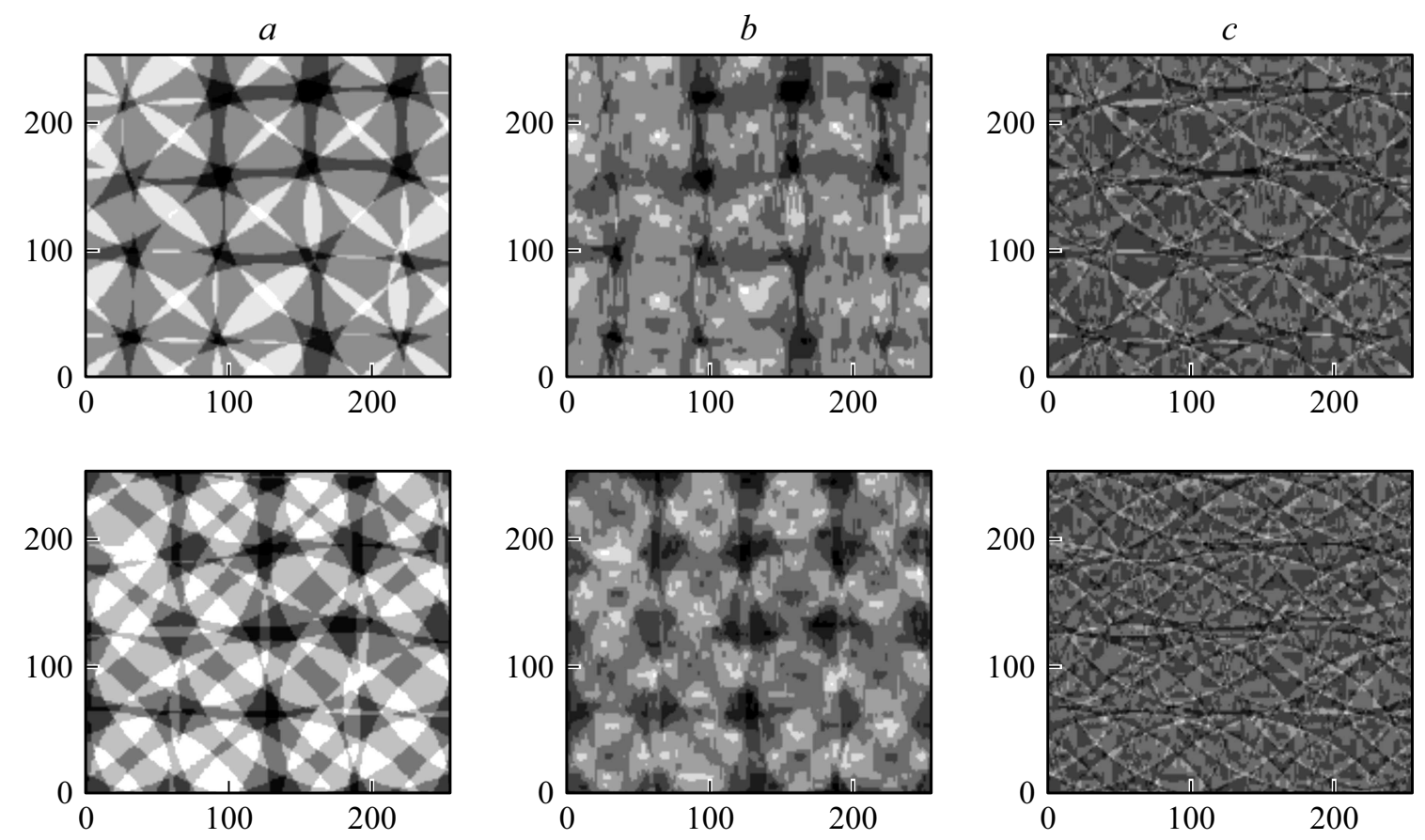

Рис. 3. Результаты моделирования квадратной решетки: $a-$ исходные изображения, полученные по формуле $(1) ; b-$ изображения, полученные в результате вейвлет-преобразования и медианного сглаживания; $c$ - изображения шума.

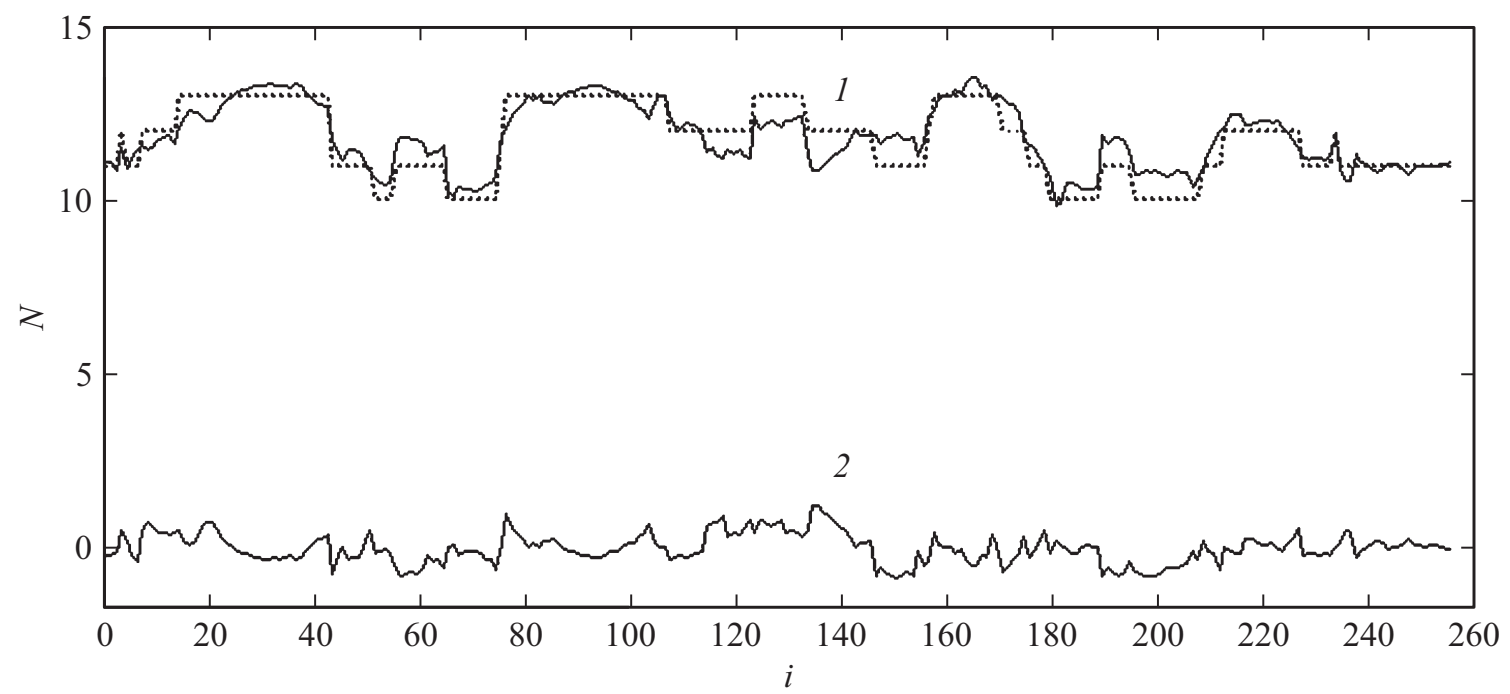

Рис. 4. Профили изображений: 1 - исходный профиль (пунктирная кривая) и результат сглаживания (сплошная кривая); $2-$ сигнал шума.

ему по величине, и $(N-1) / 2$ элементов, равных или бо́льших ему по величине. В рамках рассматриваемой задачи проводилось сравнение медианного сглаживания со сглаживанием по методу наименьших квадратов. Авторы пришли к выводу, что данные методы здесь дают приблизительно одинаковые результаты.

В целях реализации описанной выше математической модели в работе применялись программа Mathcad и ее встроенные функции. Программный модуль, выполняющий вейвлет-преобразование с ядром Добеши 4-го порядка и медианное сглаживание при $N=5$, показан на рис. 2.

\section{Результаты численного моделирования}

В настоящей работе с помощью формулы (1) строились изображения простой квадратной решетки с единичным периодом $(d=1)$. Визуализировалась решетка размером $2 \times 2$ с числом разбиений $256 \times 256$. Коор- 
динаты узлов решетки $x_{i}$ и $y_{j}$ вычислялись с учетом разброса, который формировался с помощью генератора случайных чисел. Таким образом, поверхность представляла собой искаженную квадратную решетку, узлы которой располагались случайным образом в пределах заданного отклонения с нулевым математическим ожиданием. В вычислительном эксперименте изменялись степень искажения решетки, которая составляла определенную долю от периода решетки, радиус окружности $R$ и уровень выделения спектра $\Delta$.

Под атомарным разрешением здесь понимается однозначное и отчетливое воспроизведение структуры исходной решетки на изображении. Моделирование показывает, что изображения идеальной решетки передают атомарное разрешение без неустранимых искажений, если радиус окружности превышает период решетки в десятки раз. Причем в этом случае существенная реконструкция изображений не требуется, поскольку они сохраняют регулярную структуру, соответствующую структуре решетки. Однако ситуация кардинально меняется, если $R>d$ и имеется случайный разброс в положении узлов решетки (атомов). На рис. 3 показаны результаты моделирования изображений при наличии случайного разброса величиной $2 \%$. Уровень выделения спектра $\Delta=1.5$, радиус окружности $R=1.5 d$ для верхних рисунков и $R=2 d$ для нижних рисунков. На рис. 4 показан пример получаемой функции профиля изображения вдоль одной линии сканирования.

Из рис. 3 можно видеть, что при наличии случайного разброса координат узлов увеличение радиуса $R$ принципиально не приводит к исчезновению атомарного разрешения. При этом период чередования светлых пятен, отвечающих максимумам на этих изображениях, соответствует периоду исходной решетки. Если же радиус окружности больше периода решетки и случайный разброс координат узлов превышает 5\%, то это влечет за собой исчезновение атомного контраста изображения. Моделирование также показывает, что изменение радиуса на величину $\Delta R=d / 2$ приводит к инверсии контраста, когда на изображении максимумы и минимумы меняются местами. На практике подобное явление часто наблюдается в СТМ и АСМ при многократном сканировании одного и того же участка поверхности в режимах регистрации тока или силы. По нашему мнению, данное явление может объясняться притуплением зонда или изменением общих условий измерений силы взаимодействия или туннельного тока в процессе сканирования. Явление инверсии контраста наблюдается также при моделировании контактного взаимодействия открытых однослойных и многослойных нанотрубок с поверхностью твердого тела [17]. При определенных сочетаниях периодов поверхностной структуры и радиусов нанотрубок происходит инверсия контраста изображений. Как выясняется, этот геометрический эффект вызван изменением эффективного числа атомов поверхности, попадающих в „поле видимости“ атомов углерода, локализованных на кончике нанотрубки.
Таким образом, можно сделать вывод, что асимметрия СЗМ-изображений, которая часто наблюдается экспериментально, может быть связана не только с физическими процессами в системе „зонд-образец“, но и с геометрией самой контактной зоны. В настоящей работе рассмотрены особенности формирования СЗМизображений с атомарным разрешением на примере простейшей кристаллической структуры. Однако известно, что для аморфных тел различной природы (полупроводниковых, диэлектрических, металлических) всегда имеется универсальный пространственный масштаб $\sim 1 \mathrm{~nm}$ - своеобразный параметр порядка, который играет такую же важную роль, как элементарная ячейка для кристаллов [18]. Неоднородности в данных материалах представляют собой не единичные образования и аналоги дефектов в кристаллах, а фрагменты, из которых целиком построены аморфные тела. Применение СЗМ и предложенной в настоящей работе методики моделирования может играть конструктивную роль при исследовании структуры указанных неоднородностей.

\section{Заключение}

В настоящей работе с помощью математического моделирования показано, что основными факторами, влияющими на атомарное разрешение в СЗМ, являются размер контактной зоны и наличие дефектов кристаллической структуры. В данном случае, разумеется, не имеются в виду конструкционные особенности СЗМ, механический дрейф, шумы электрических схем и загрязнения исследуемой поверхности. Если исследуемая поверхность не содержит дефекты, то для получения истинного атомарного разрешения линейный размер контактной зоны может в десятки и даже в сотни раз превышать межатомное расстояние. Все это дает ключ к объяснению известного парадокса атомарного разрешения в СЗМ при использовании тупых зондов.

Моделирование выявляет высокую эффективность вейвлет-преобразования при обработке модельных СЗМизображений, что объясняется основными свойствами базовых вейвлетов, а именно их автомодельностью и ограниченностью. Вейвлет-преобразование может использоваться для анализа фрактальной структуры профилей поверхностей, что представляет собой чрезвычайно актуальную задачу [19]. Коэффициенты $W(a, b)$ отображают иерархическую структуру профиля: каждому масштабному уровню соответствует локальный экстремум в спектре. Фрактальная размерность соответствующего сигнала может быть вычислена по коэффициентам вейвлет-преобразования как предел (при стремлении к нулю масштаба) отношения $\ln N(a) / \ln a$, где $N(a)-$ число локальных максимумов в спектре $[13,19]$.

Работа выполнена в рамках программы „Leading-Talent Program of Guangdong Province“ (№ 00201502). 


\section{Список литературы}

[1] Davies J.H., Hyldgaard P., Hershfield S., Wilkins J.W. // Phys. Rev. B. 1992. Vol. 46. N 15. P. 9620-9633.

[2] Mizes H.A., Park S., Harrison W.A. // Phys. Rev. B. 1987. Vol. 36. N 8. P. 4491-4494.

[3] Flatte M.E., Byers J.M. // Phys. Rev. B. 1996. Vol. 53. N 16. P. 10536-10539.

[4] Рехвиашвили С.Ш., Розенберг Б.А., Дремов В.В. // Письма в ЖЭТФ. 2008. Т. 88. № 11. С. 805-809.

[5] Abraham F.F., Batra I.P., Ciraci S. // Phys. Rev. Lett. 1988. Vol. 60 N 13. P. 1314-1317.

[6] Kobayashi K., Tsukada M. // J. Vac. Sci. Technol. A. 1990. Vol. 8. P. 170.

[7] Isshiki N., Kobayashi K., Tsukada M. // Surface Science. 1990. Vol. 238. N 1-3. P. L439-L445.

[8] Watanaba S., Aono M., Tsukada M. // Phys. Rev. B. 1991. Vol. 44. N 15. P. 8330-8333.

[9] Tekman E., Ciraci S. // J. Phys.: Condens. Mater. 1991. Vol. 3. N 6. P. 2613-2619.

[10] Покропивный А.В., Покропивный В.В., Скороход В.В. // ЖТФ. 1997. Т. 67. № 12. С. 70-75.

[11] Рехвиашвили С.Ш. // Математическое моделирование. 2003. T. 15. № 2. C. 62-68.

[12] Рехвиашвили С.Ш. // Письма в ЖТФ. 2002. Т. 28. № 6. C. $46-50$.

[13] Астафбева H.М. // УФН. 1996. Т. 166. № 11. С. 1145-1170.

[14] Дьяконов В.П. Вейвлеты. От теории к практике. М.: Солон-Р, 2002. 448 c.

[15] Прэтт У. Цифровая обработка изображений. Кн. 2. М.: Мир, 1982. 480 c.

[16] Миронов В.Л. Основы сканирующей зондовой микроскопии. М.: Техносфера, 2005. 144 с.

[17] Дедков Г.В., Рехвиашвили С.Ш. // ЖТФ. 1999. Т. 69. № 8. C. 124-127.

[18] Малиновский В.К. // ФТТ. 1999. Т. 41. № 5. С. 805-808.

[19] Потапов А.А. Фракталы в радиофизике и радиолокации: Топология выборки. Изд. 2-е, перераб. и доп. М.: Университетская книга, 2005. 848 с. 